\title{
Exercising control over bipolar disorder
}

\author{
Gin S Malhi, ${ }^{1,2,3}$ Yulisha Byrow ${ }^{1,2,3}$
}

${ }^{1}$ Academic Department of Psychiatry, Kolling Institute, Northern Sydney Local Health District, St Leonards, New South Wales, Australia;

${ }^{2}$ Sydney Medical School Northern, University of Sydney, Sydney, New South Wales, Australia; ${ }^{3}$ CADE Clinic, Royal North Shore Hospital, Northern Sydney Local Health District, St Leonards, New South Wales, Australia

Correspondence to Professor Gin S Malhi; gin.malhi@sydney.edu.au

\begin{abstract}
Following extensive research exercise has emerged as an effective treatment for major depressive disorder, and it is now a recognised therapy alongside other interventions. In contrast, there is a paucity of research examining the therapeutic effects of exercise for those with bipolar disorder. Given that dysfunctional reward processing is central to bipolar disorder, research suggests that exercise can perhaps be framed as a reward-related event that may have the potential to precipitate a manic episode. The behavioural activation system (BAS) is a neurobehavioural system that is associated with responding to reward and provides an appropriate framework to theoretically examine and better understand the effects of exercise treatment on bipolar disorder. This article discusses recent research findings and provides an overview of the extant literature related to the neurobiological underpinnings of BAS and exercise as they relate to bipolar disorder. This is important clinically because depending on mood state in bipolar disorder, we postulate that exercise could be either beneficial or deleterious with positive or negative effects on the illness. Clearly, this complicates the evaluation of exercise as a potential treatment in terms of identifying its optimal characteristics in this population.
\end{abstract}

\section{INTRODUCTION}

Behavioural activation is a commonly used psychological treatment for major depressive disorder (MDD) that broadly refers to the development of activity scheduling and also targets cognitive processes that are linked to avoidance. ${ }^{1}$ Research findings suggest that for MDD behavioural activation is generally an effective therapeutic intervention, ${ }^{2}$ with some studies suggesting that it is equally as effective as cognitive therapies or a combination of cognitive and behavioural activation therapies. $^{3}$ Behavioural activation is particularly relevant to depressive episodes, where individuals experience difficulty engaging in physical activity because of psychomotor retardation; feeling slowed down physically and mentally. The converse is true in mania where individuals usually experience increased energy and activity levels. This may partly explain why behavioural activation has been extensively studied in relation to $\mathrm{MDD}$, but there is little research as yet, investigating its effectiveness in treating bipolar disorder. Therefore, in this article, we consider the emerging research findings examining the therapeutic effectiveness of exercise as a behavioural activation strategy for those with bipolar disorder. Furthermore, we propose the use of a neurobehavioural model for understanding the effects of this treatment strategy on depressive and manic mood states. Pertinent literature was identified using Google Scholar and PubMed, with relevant search terms - such as 'bipolar disorder', 'exercise' and acceptable variations of these (update: June 2016). Given the paucity of the literature, the reference lists of all identified papers were then inspected for further potential resources with particular attention paid to more recent research findings.

\section{DEPRESSION AND EXERCISE}

Depression is associated with many physical health risks, including heightened risk for cardiovascular disease and type II diabetes. Behavioural activation involves scheduling activities that are in line with patients' values; therefore, there is a distinction between physical activity or exercise in general and exercise as a component of behavioural activation. Depending on the severity of illness exercise per se is a recommended therapy for $\mathrm{MDD}^{4}$ either as an adjunctive treatment ${ }^{5} 6$ or in some mild-to-moderate cases as a first-line treatment option or a second-line treatment option. ${ }^{5}$ Studies examining the therapeutic benefits of exercise in depressed patients suggest many benefits such as improved physical health, lower depressive symptoms and better psychological well-being. ${ }^{7}$ Results from a recent Cochrane review, defining exercise as 'planned, structured and repetitive bodily movement done to improve or maintain one or more components of physical fitness', indicate a moderate association (reported effect size of -0.62 when comparing exercise with no treatment or control intervention) between exercise and reduced depressive symptoms in adults diagnosed with depression. However, studies examining the long-term effects or highquality trials have demonstrated only small associations and nonsignificant associations, respectively. ${ }^{8}$ More recently, studies have investigated the most effective type and dose of exercise in treating depression. The findings from a systematic review suggest that physical activity should be individually tailored, last a minimum of $30 \mathrm{~min}$, ideally be conducted under supervised conditions and performed at least three times a week. However, these conclusions are somewhat constrained by the relatively few studies that met inclusion criteria (12 studies). ${ }^{9}$ Therefore, it seems the benefits of exercise within the context of behavioural activation are beneficial to those with depressive symptoms, however the optimal parameters of this treatment (eg, frequency, duration, type of exercise) are yet to be determined.

\section{BIPOLAR DISORDER}

In contrast to the literature on MDD, there is a notable lack of research examining the effects of exercise on those with bipolar disorder. Furthermore, the findings from the few studies that have been conducted in bipolar disorder have been limited by issues of research methodology such as small sample sizes, inadequate control conditions and scarce measurement of current mood state. For example, $\mathrm{Ng}$ and colleagues examined the effects of a walking group on 14 bipolar inpatients with regular attendance. Those who participated in the walking group reported significantly lower levels of depression, stress, and anxiety. ${ }^{10}$ In a proof of concept study, Sylvia et $a l^{11}$ evaluated the feasibility of an adjunctive 20 week cognitive-behavioural therapy based nutrition, exercise and wellness treatment for obese patients with bipolar disorder. Although the sample size in this study was extremely small $(n=5)$, the preliminary findings suggest that this programme reduced depressive symptoms in these patients. Another study examined the effectiveness of the 'In SHAPE' programme in a 'mixed' sample of patients presenting predominantly with schizophrenia, MDD and bipolar disorder and found that while there was no significant effect on depressive symptoms, a positive effect on mental health functioning and negative symptoms was reported..$^{12}$ One of the reasons for the paucity of research in bipolar disorder could be the concern that exercise may trigger a manic episode. Central to bipolar disorder is the dysfunctional processing of reward, for example, anhedonia during depression and risk-taking/pleasureseeking during mania. Research findings examining potential triggers of 
hypo/manic episodes seem to corroborate this theory and have shown that activities involving 'recreational stimulant use, partying all night and periods of personal growth', for example, precipitated manic episodes while 'stressful life events, general stress, fatigue and decreased exercise' more often preceded depressive episodes. ${ }^{13}$ Some research groups have conceptualised the effects of exercise on bipolar symptoms as a 'double-edged sword'. ${ }^{14}$ Their qualitative analysis highlights several important themes to consider before implementing an exercise treatment for bipolar disorder. Their findings suggest that exercise may be beneficial for those with bipolar disorder, when aiming to reduce symptom severity and protect against exacerbations. To achieve this, it is suggested that an exercise intervention should be flexible, not necessarily administered during severe mood episodes, as well as modifying the routine during different mood episodes. The study also highlights that exercise intensity and type of exercise are key considerations that may differ depending on the patient's current mood state. For example, many participants perceived more benefit from lighter exercise routines during hypomania, whereas others reported that rhythmic exercises leg, running, cycling, rowing, swimming) were of more benefit. Most importantly, the authors emphasise that practitioners should be mindful of potential benefits and harm that may arise from exercise interventions during hypomania. Risks include the potential to exercise excessively thereby accelerating activation in hypomanic individuals. ${ }^{14}$

\section{NEUROBIOLOGICAL FRAMEWORK FOR UNDERSTANDING THERAPEUTIC EFFECTS OF EXERCISE}

In order to understand why exercise might trigger a manic episode, it is important to consider the neurobiological mechanisms underlying the effects of exercise on those with bipolar disorder. Originating in personality research, the behavioural activation (or approach) system (BAS) is a neurobehavioural system implicated in responding to reward or incentives. $^{15}$ In bipolar disorder, the BAS is thought to be dysfunctional and hyper-responsive, such that the system is activated during mania and deactivated in depression, and this is reflected in increased reward seeking behaviour and anhedonia, respectively. ${ }^{13} 16$ In other words, events related to BAS activation in bipolar disorder can potentially precipitate a (hypo)manic episode. Hence, exercise can be viewed as a BAS activating event that involves the potential to attain reward. ${ }^{16}$ Similarly, events that are specifically related to BAS deactivation such as loss of or failure to attain reward can precipitate a depressive episode. ${ }^{17} 18$ Therefore, adopting a BAS framework to investigate the association between exercise and bipolar disorder seems logical and coincides with reports by Wright et $a l^{14}$ that bipolar patients may have negative experiences when exercising during hypo/manic episodes. Research shows that those with bipolar disorder report higher levels of BAS sensitivity (measured using self-report questionnaires) and have greater responsiveness towards reward on behavioural tasks. ${ }^{17} 18$ Furthermore, participants with high BAS sensitivity are more likely to develop bipolar or related disorders over $\sim 1$ year than those with moderate BAS sensitivity. ${ }^{19}$

Although the processing of reward is not limited to a single biological process, key brain networks implicated include the mesocorticolimbic pathways (including connections within ventral and dorsal striatal brain regions). ${ }^{20}$ These neural brain regions and their connections are rich in dopamine receptors and have been shown to be dysfunctional in individuals with major depression and bipolar disorder. ${ }^{21}$ Similar networks and brain regions have been implicated as central to the BAS (ie, orbitofrontal cortex, nucleus accumbens and amygdala). ${ }^{22}$ Urosevic et $a^{22}$ examined participants ranging from 9 to 23 years in age over a 2-year period and found that the differences in volume of the nucleus accumbens and medial orbitofrontal cortex, measured at baseline, predicted increases in reward sensitivity at follow-up. More recent research findings suggest that $\mu$-opoid receptor availability in the prefrontal, cingulate and insular cortices and subcortical regions including the ventral striatum, amygdala and brainstem was associated with greater BAS sensitivity. ${ }^{23}$

\section{NEUROBIOLOGICAL EFFECTS OF EXERCISE}

A recent systematic review highlights that there is a lack of research examining neural activity and brain structure in relation to exercise and major depression with only three eligible studies examining this hypothesis and no studies examining the acute effects of exercise on neural activity. ${ }^{24}$ For example, Krogh et $a l^{25}$ found no changes in hippocampal volume associated with exercise, although there were no significant effects of exercise on depressive symptoms observed in this study. Instead, the review findings suggest that acute exercise increases atrial natriuretic peptide, brain natriuretic peptide, copepetin and growth hormone in those with MDD, all of which are physiological factors implicated in managing the neuroendocrine system. Research studies investigating healthy individuals indicate that exercise is associated with increases in brain-derived neurotrophic factor (BDNF), the expression of which supports neurogenesis. ${ }^{26}$ Therefore, the authors propose that exercise may be beneficial for those with bipolar disorder by producing higher levels of BDNF and neurogenesis, which in turn influences allostasis (the process whereby neuromodulators are released as a response to a stressful event) by reducing allostatic load; in other words enhancing the ability to cope with stress. However, findings from the systematic review conducted by Schuch et $a^{24}$ did not find any support for this proposal in samples with MDD. But it is important to note that the patients in the reviewed studies were all prescribed adjunctive antidepressant treatment, and this may have confounded results.

In terms of bipolar disorder, there has been some research suggesting that exercise may influence executive functioning in these samples. For example, a recent study examining the effects of acute exercise on adolescents completing a measure of executive functioning (go/no-go task) has shown that acute aerobic exercise is able to influence deficits in deactivating attention and activating inhibition. ${ }^{27}$ This line of inquiry is important to understand the potential therapeutic efficacy of exercise for bipolar disorder. Taken together these findings suggest that the BAS is not the only dysfunctional biological circuit in bipolar disorder. While a BAS framework accounts for the positive effects of exercise on anhedonia and the negative effects of exercise on hypo/ mania, it does not adequately explain the potential positive effects of exercise on hypo/mania. Some patients report that exercise during mania is helpful to expend excess energy and restore a sense of structure or 'inherent rhythm' to otherwise dysregulated internal states. ${ }^{14}$ Although based on qualitative self-report, these findings are important and suggest that exercise may also affect important biological regulatory mechanisms that are dysfunctional in bipolar disorder (see figure 1).

\section{FUTURE DIRECTIONS}

Given the lack of research investigating the therapeutic effects of exercise on those with bipolar disorder, it remains difficult to evaluate its efficacy and optimal translation into clinical implementation. From existing findings, it appears that those with bipolar disorder have a hypersensitive BAS, and neurobiological findings suggest that reward processing in particular is impaired in bipolar disorder. Collectively, these findings suggest that exercise may have positive and negative effects that are dependent on current mood state. It would be useful for future investigations to examine the effects of exercise on hypo/ manic, depressed and euthymic individuals, varying its parameters (such as the type, duration and frequency of exercise). A particularly interesting question is whether these factors differentially impact bipolar individuals depending on their current mood state? It is clear that exercise is of benefit to those with depression and may be of benefit in certain circumstances to those with hypo(mania). Disentangling the specific effects of exercise on mood state is a critical first step in understanding whether non-pharmacological interventions, such as exercise, will be beneficial for treating bipolar disorder. 
Figure 1 The potential effects of exercise on the BAS in bipolar disorder. The BAS is thought to be overly sensitive in bipolar disorder. Thus, events related to gaining reward, for example, exercise, are thought to increase BAS activation (red arrow). In the context of a depressive episode (blue circle), increased BAS activation is likely to improve depressive symptoms such as anhedonia. However, in the context of a manic episode (green circle), increases in BAS activation could worsen hypo/ manic symptoms. The orange arrow represents the potential positive effects of exercise on hypo/manic symptoms that perhaps occur by entraining asynchronous systems and re-establishing control over dysfunctional biological mechanisms. BAS, behavioural activation system.

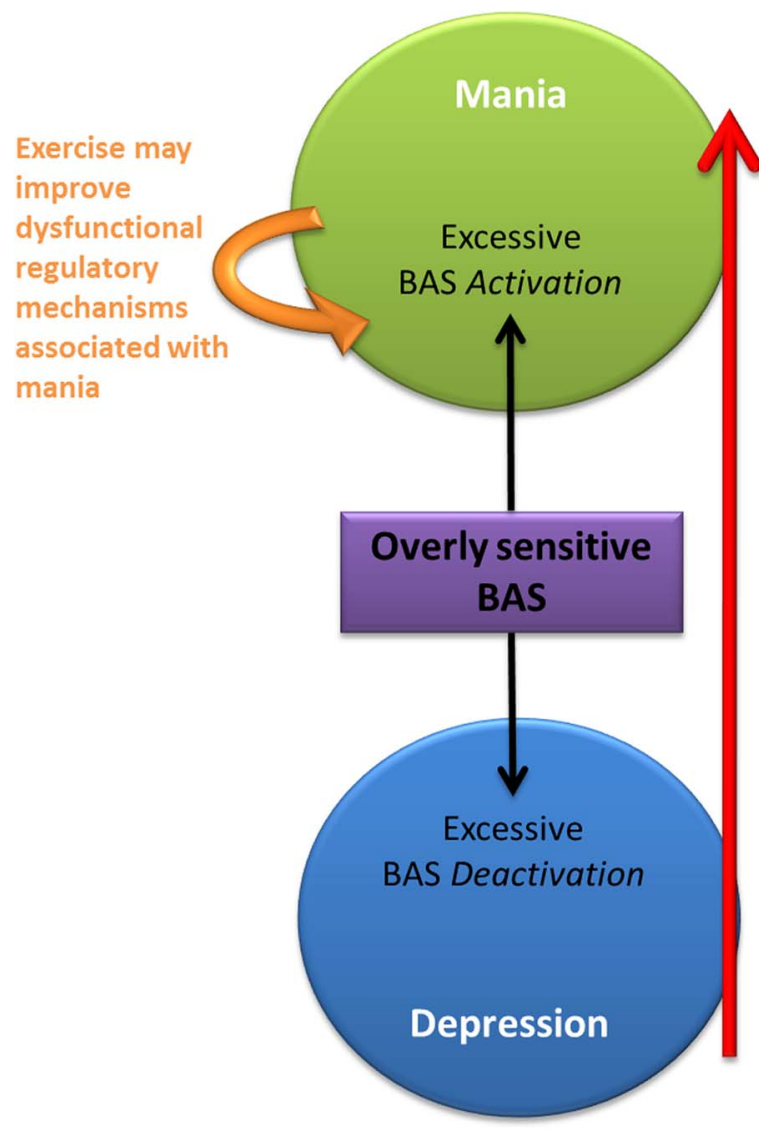

Increased BAS activation may worsen hypo/manic symptoms

Exercise increases BAS activation

Increased BAS activation improves depressive symptoms

Competing interests None declared.

Provenance and peer review Not commissioned; externally peer reviewed. doi:10.1136/eb-2016-102430

Received 8 July 2016; Revised 15 August 2016; Accepted 17 August 2016

\section{REFERENCES}

1. Martell C, Addis M, Jacobson N. Depression in context: strategies for guided action. New York: WW Norton; 2001:224.

2. Ekers D, Webster L, Van Straten A, et al. Behavioural activation for depression; an update of meta-analysis of effectiveness and sub group analysis. PLoS One 2014;9: e100100.

3. Jacobson N, Dobson K, Truax P. A component analysis of cognitive-behavioral treatment for depression. J Consult Clin Psychol 1996;64:295-304.

4. Malhi GS, Bassett D, Boyce P, et al. Royal Australian and New Zealand College of Pcyhiatrists clinical practice guidelines for mood disorders. Aust N Z J Psychiatry 2015;49:1087-206.

5. Ravindran A V, Balneaves LG, Faulkner G, et al., CANMAT Depression Work Group. Canadian Network for Mood and Anxiety Treatments (CANMAT) 2016 Clinical Guidelines for the Management of Adults with Major Depressive Disorder: Section 5. Complementary and alternative medicine treatments. Can J Psychiatry 2016;61:576-87.

6. Cleare A, Pariante $\mathrm{CM}$, Young $\mathrm{AH}$, et al., Members of the Consensus Meeting. Evidence-based guidelines for treating depressive disorders with antidepressants: a revision of the 2000 British Association for Psychopharmacology guidelines. J Psychopharmacol 2015;29:459-525.

7. Schuch FB, Vasconcelos-Moreno MP, Borowsky C, et al. Exercise and severe major depression: effect on symptom severity and quality of life at discharge in an inpatient cohort. J Psychiatr Res 2015;61:25-32.

8. Cooney GM, Dwan K, Greig CA, et al. Exercise for depression [review]. Cochrane Database Syst Rev 2013;9:CD004366.

9. Nyström MBT, Neely G, Hassmén P, et al. Treating major depression with physical activity: a systematic overview with recommendations. Cogn Behav Ther 2015;44:341-52.

10. $\mathbf{N g ~ F}$, Dodd S, Berk M. The effects of physical activity in the acute treatment of bipolar disorder: a pilot study. J Affect Disord 2007;101:259-62.

11. Sylvia LG, Salcedo S, Bernstein EE, et al. Nutrition, exercise, and wellness treatment in bipolar disorder: proof of concept for a consolidated intervention. Int $J$ bipolar Disord 2013;1:24.

12. Van Citters AD, Pratt SI, Jue K, et al. A pilot evaluation of the in SHAPE individualized health promotion intervention for adults with mental illness. Community Ment Health J 2010;46:540-52.
13. Proudfoot $\mathbf{J}$, Whitton A, Parker G, et al. Triggers of mania and depression in young adults with bipolar disorder. J Affect Disord 2012;143:196-202.

14. Wright $\mathbf{K}$, Armstrong T, Taylor A, et al. 'It's a double edged sword': a qualitative analysis of the experiences of exercise amongst people with bipolar disorder. $J$ Affect Disord 2012;136:634-42.

15. Gray $\mathbf{J}$. The psychophysiological basis of introversion-extraversion: a modification of Eysenck's theory. In: Nebylitsyn V, Gray J, eds. The biological basis of individual behavior. New York: Academic, 1972:182-205.

16. Thomson D, Turner A, Lauder $\mathrm{S}$, et al. A brief review of exercise, bipolar disorder, and mechanistic pathways. Front Psychol 2015;6:1-10.

17. Urosevic $\mathbf{S}$, Abramson $L Y$, Harmon-Jones $E$, et al. Dysregulation of the behavioral approach system (BAS) in bipolar spectrum disorders: review of theory and evidence. Clin Psychol Rev 2008;28:1188-205.

18. Alloy LB, Abramson LY. The Role of the Behavioral Approach System (BAS) in bipolar spectrum disorders. Curr Dir Psychol Sci 2010;19:189-94.

19. Alloy LB, Bender RE, Whitehouse WG, et al. High Behavioral Approach System (BAS) sensitivity, reward responsiveness, and goal-striving predict first onset of bipolar spectrum disorders: a prospective behavioral high-risk design. J Abnorm Psychol 2012;121:339-51.

20. Whitton AE, Treadway MT, Pizzagalli DA. Reward processing dysfunction in major depression, bipolar disorder and schizophrenia. Curr Opin Psychiatry 2015;28:7-12.

21. Malhi GS, Byrow Y, Fritz K, et al. Mood disorders: neurocognitive models. Bipolar Disord 2015;17:3-20.

22. Urošević $\mathbf{S}$, Collins $\mathrm{P}$, Muetzel $\mathrm{R}$, et al. Longitudinal changes in behavioral approach system sensitivity and brain structures involved in reward processing during adolescence. Dev Psychol 2012;48:1488-500.

23. Karjalainen T, Tuominen L, Manninen S, et al. Behavioural-activation-system sensitivity is associated with cerebral $\mu$-opioid receptor availability. Soc Cogn Affect Neurosci 2016;11:1310-16.

24. Schuch FB, Deslandes AC, Stubbs B, et al. Neurobiological effects of exercise on major depressive disorder: a systematic review. Neurosci Biobehav Rev 2016;61:1-11.

25. Krogh J, Rostrup E, Thomsen C, et al. The effect of exercise on hippocampal volume and neurotrophines in patients with major depression-a randomized clinical trial. J Affect Disord 2014;165:24-30.

26. Sylvia LG, Ametrano RM, Nierenberg AA. Exercise treatment for bipolar disorder: potential mechanisms of action mediated through increased neurogenesis and decreased allostatic load. Psychother Psychosom 2010;79:87-96.

27. Metcalfe AWS, Maclntosh BJ, Scavone A, et al. Effects of acute aerobic exercise on neural correlates of attention and inhibition in adolescents with bipolar disorder. Trans/ Psychiatry 2016;6:e814 